\title{
Inspection of Spray on Foam Insulation (SOFI) Using Microwave and Millimeter Wave Synthetic Aperture Focusing and Holography
}

\author{
J.T. Case ${ }^{1}$, F.L. Hepburn ${ }^{2}$, R. Zoughi ${ }^{1}$ \\ ${ }^{1}$ Applied Microwave Nondestructive Testing Laboratory (amntl) \\ Electrical and Computer Engineering Department \\ University of Missouri-Rolla, Rolla, MO 65409, USA \\ Email: zoughir@umr.edu, URL: http://www.ece.umr.edu/amntl/ \\ ${ }^{2}$ NASA George C. Marshall Space Flight Center \\ Huntsville, AL 35812, USA
}

\begin{abstract}
The Space Shuttle Columbia's catastrophic failure is thought to have been caused by a dislodged piece of external tank spray on foam insulation (SOFI) striking and significantly damaging the left wing of the orbiter, which may have been due to a flawed section of SOFI. Microwave and millimeter wave nondestructive evaluation (NDE) methods have shown great potential detecting anomalies in SOFI such as small air voids using $a$ horn and lens in a (real) focused configuration. Synthetic focusing methods may also be used to detect air voids in SOFI and may additionally offer the ability to locate the defect in three dimensions. To this end, two different methods were investigated; namely, frequency domain synthetic aperture focusing technique (FD-SAFT) and wide-band microwave holography. To illustrate the performance of these methods they were applied to two different SOFI samples. The results of these investigations demonstrate the capabilities of these methods for SOFI inspection.
\end{abstract}

Keywords - microwave, millimeter wave, nondestructive testing, foam, SOFI.

\section{INTRODUCTION}

The Space Shuttle Columbia's catastrophic failure is thought to have been caused by a dislodged piece of external tank spray on foam insulation (SOFI) striking the left wing of the orbiter causing significant damage to some of the reinforced carbon/carbon leading edge wing panels [1]. Consequently, an effective NDE method for detecting small air voids and other defects has been sought for this purpose. Microwave and millimeter wave nondestructive evaluation methods have shown great potential for inspecting SOFI for the purpose of detecting anomalies such as small air voids that may cause separation of the SOFI from the external tank during a launch [2,3]. These methods are capable of producing relatively high-resolution images of the interior of SOFI. These methods utilized antennas or lenses for focusing the microwave or millimeter wave signal and hence are referred to as real focused techniques. However, there are some advantages to using synthetic focusing methods for SOFI inspection including reduced probe size, the ability to determine depth of a defect from multiple views or swept frequency measurements, and the ability to slice two dimensional images from a volumetric dataset. To this end, synthetic aperture focusing techniques (SAFT) were first implemented for this purpose and later microwave holography was used [4-7].

This paper presents the results of this investigation using frequency domain synthetic aperture focusing technique (FDSAFT) and wide-band microwave holography methods illustrating their potential capabilities for inspecting the Space Shuttle's SOFI at millimeter wave frequencies. These methods were applied to scanned data sets of two different SOFI configurations: A) three arrangements of SOFI slab panels containing $25 \mathrm{~mm}$-diameter holes of varying depths and B) a SOFI test panel containing 20 different holes of varying depths and diameters. The results demonstrate the promise of these image processing methods in particular when they are later applied at much higher frequencies.

\section{APPROACH AND PANEL SPECIFICATION}

Microwave NDE methods are particularly suitable for SOFI inspection since the wavelength is large enough to consider the medium as a homogeneous dielectric since SOFI is comprised of very small air bubbles contained in low loss (dielectric) polymer. Also, the wavelength is small enough to provide satisfactory image resolution. The SOFI samples were raster scanned by the automated movement of a rectangular waveguide probe over the sample with a separation (i.e., liftoff) of $10 \mathrm{~mm}$, as shown in Figure 1 . Measurements were performed at K-band $(18-26.5 \mathrm{GHz})$. The sampling increment was $2 \mathrm{~mm}$ or roughly half of the narrow dimension of the waveguide probe. The sampling points were limited to one plane referred to as the measurement plane and corresponding to $z=0$. Data was acquired using the Agilent E8361A PNA Series Network Analyzer where the magnitude, $\Pi \Gamma$, and phase, $\phi$, of the microwave reflection coefficient at the aperture of the waveguide probe were recorded for every data point. For this measurement setup, internal (via the PNA) coherent subtraction of the reflection at the waveguide-to-air boundary was readily possible. However, the mean of the reflection coefficient was also subtracted to effectively subtract the contribution of the flat metal substrate rendering small scatterers more pronounced. 


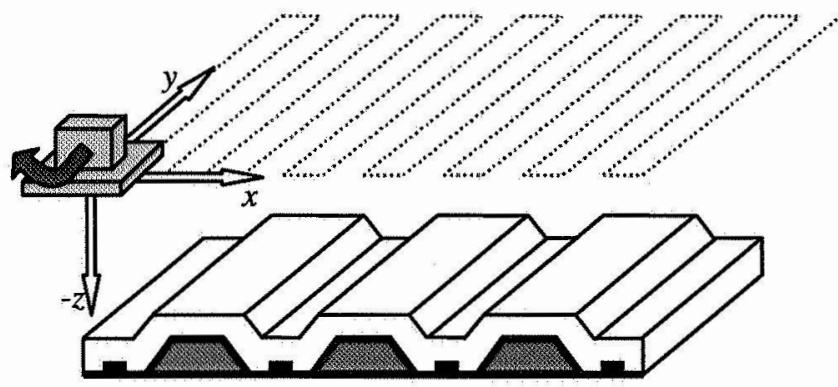

Fig. 1. Illustration of scanning procedure with open-ended waveguide probe over a SOFI sample.

\section{A. SOFI Slab Panels}

The SOFI slab panels consisted of three arrangements of two $70 \mathrm{~mm}$-thick panels: one with five $25 \mathrm{~mm}$-diameter flatbottom holes and another without (called the blank panel). 25 mm-diameter holes were thought to provide a relatively strong indication at $\mathrm{K}$-band. The holes had been drilled right to left to the following depths: $3 \mathrm{~mm}, 6 \mathrm{~mm}, 13 \mathrm{~mm}, 19 \mathrm{~mm}$, and $25 \mathrm{~mm}$, as shown in Figure 2. The panels were arranged in three different configurations: 1) panel with holes face down and backed by an aluminum substrate (Figure $2 b$ ), 2) panel with holes face down and backed by a blank panel and substrate (Figure 2c), and 3) panel with holes face down and sandwiched by the blank panel on top and the substrate on the bottom (Figure $2 \mathrm{~d}$ ).

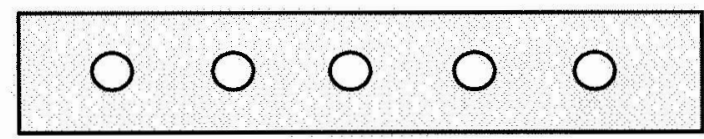

(a)

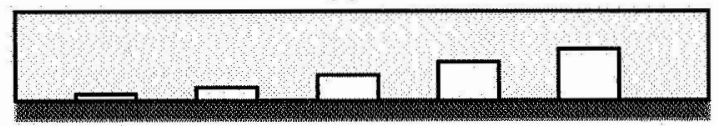

(b)

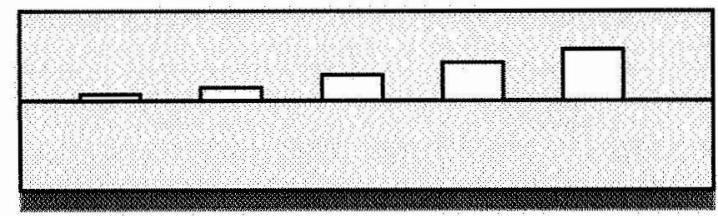

(c)

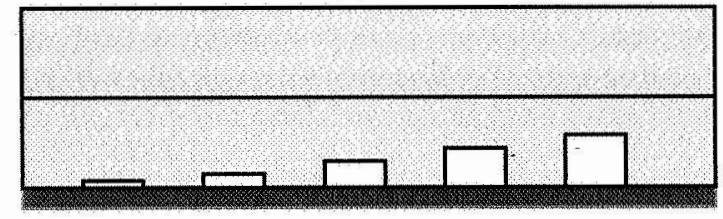

(d)

Fig 2. Flat-bottom hole schematic: (a) top view, (b) case 1, (c) case 2, (d) case 3.
The purpose of the first two arrangements was to analyze the behavior of moving the substrate away while retaining the height of the holes. It was expected that the results of these two arrangements would be similar if the holes themselves were detected. The purpose of arrangements 1 and 3 were to observe moving the holes away from the measurement plane. This can significantly reduce the strength of the signal associated with the holes until they are undetectable.

\section{B. Multiple Flat-Bottom Hole SOFI Test Panel}

This panel was constructed to investigate the potential resolution and the capability of detecting of air voids in SOFI with a fixed substrate height. The panel measured $305 \mathrm{~mm}$ by $305 \mathrm{~mm}$ and $76 \mathrm{~mm}$ thick. The flat-bottom holes in the sample were produced with diameters ranging from $3 \mathrm{~mm}$ to $25 \mathrm{~mm}$ and depths ranging from $3 \mathrm{~mm}$ to $19 \mathrm{~mm}$, as shown in Figure 3. The strength of the reflected signal from the holes is expected to decrease for decreasing hole diameter and decreasing hole depth. From these results it may be possible to determine the minimum detectable defect size for an air void in SOFI for a given method of image processing, frequency of operation, antenna type, displacement of substrate to the measurement plane, and measurement plane size.

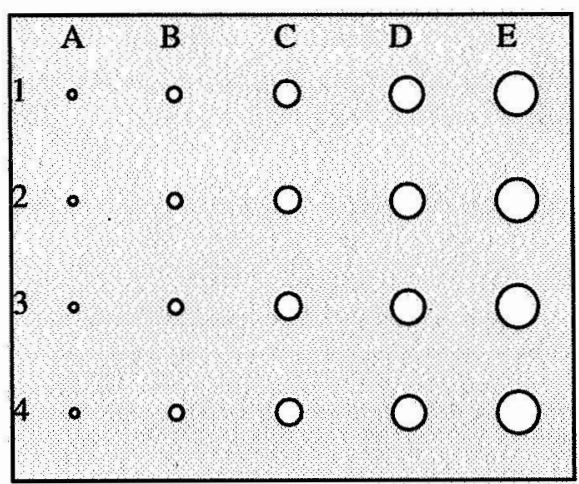

(a)

1

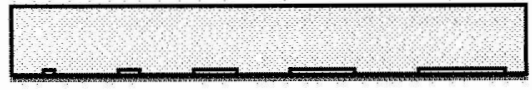

2

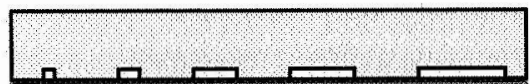

3

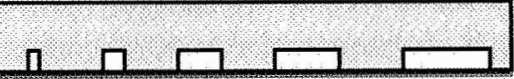

4

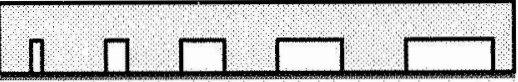

(b)

Fig 3. Schematic of multiple flat-bottom hole SOFI panel: (a) top view, (b) bottom views for each row. 
The schematic of the sample is shown in Figure 3 where rows labeled 1 through 4 determine hole depths and columns labeled A through $\mathrm{E}$ determine hole diameters. The hole depths are: 1) $3 \mathrm{~mm}$, 2) $6 \mathrm{~mm}, 3$ ) $13 \mathrm{~mm}$, and 4) $19 \mathrm{~mm}$. The hole diameters are: A) $3 \mathrm{~mm}$, B) $6 \mathrm{~mm}$, C) $13 \mathrm{~mm}$, D) 19 $\mathrm{mm}$, and E) $25 \mathrm{~mm}$.

\section{METHODS}

After the full vector microwave reflection coefficient was measured at discrete locations on the measurement plane as described earlier, the raster image data was processed using two image processing methods; namely, FD-SAFT and wideband microwave holography $[4,6]$.

For calculation purposes, three assumptions are made. First, the sample under test is assumed to not disperse or depolarize the incident wave. Secondly, it is assumed that only single reflections occur and no multiple reflections exist. Lastly, although we seek to detect the small reflection between the air and SOFI boundary, the wave traveling through the SOFI is assumed not to delay significantly since the dielectric properties of SOFI and air are so similar. This last assumption allows us to use the existing algorithms with no modification regarding traveling waves through media.

\section{A. Narrow-Band FD-SAFT}

FD-SAFT stands for synthetic aperture focusing technique applied in the frequency domain [4]. The derivation pursued was L.J. Busse's narrow-band version in which the data is assumed to be single frequency and is based on angular spectrum decomposition [7]. The raw data is measured at the plane $z=0$ and is contained in:

$$
g(x, y: z=0)
$$

such that:

$$
0 \leq x \leq x_{\max } \text { and } 0 \leq y \leq y_{\max }
$$

and such that $g$ is sampled at discrete locations in $x$ and $y$. Subsequently, this must be decomposed onto a plane wave spectrum using the 2D Fast Fourier transform:

$$
G\left(k_{x}, k_{y}: z=0\right)=F F T_{2 D}\{g(x, y: z=0)\}
$$

The ranges for $k_{x}$ and $k_{y}$ are centered about zero, which is required for the backward wave propagator. Next, the data is propagated to the plane of $\mathrm{z}=-h$ so that the sample under test is brought into focus. This is done by using the two-way backward wave propagator:

$$
B(z, \omega)=\exp \left[j 2 k z\left(1-\left(k_{x} / 2 k\right)^{2}-\left(k_{y} / 2 k\right)^{2}\right)\right]
$$

where $\omega$ is the radial frequency and $k=\omega / c$. The intermediate quantity, $G^{\prime}$, after applying the backward wave propagator is defined as:

$$
G^{\prime}\left(k_{x}, k_{y}: z=-h\right)=G\left(k_{x}, k_{y}: z=0\right) \times B(z=-h, \omega)
$$

The last step is to project the data back to real space using the inverse 2D Fast Fourier transform:

$$
s(x, y: z=-h)=F F T_{2 D}^{-1}\left\{G^{\prime}\left(k_{x}, k_{y}: z=-h\right)\right\}
$$

The processed data, $s(x, y: z=-h)$, is a single frequency high-resolution focused image at height $z=-h$. The spatial resolution of this image is roughly half of the dimension of the antenna aperture similar to a focused synthetic aperture radar (SAR) [8]. A more accurate definition of the spatial resolution is:

$$
\delta_{x} \approx \frac{\lambda}{4 \sin \left(\theta_{b} / 2\right)}
$$

where $\lambda$ is the wavelength of operation and $\theta_{b}$ is either the full beamwidth of the microwave probe or the angle subtended by the measurement plane, whichever is less [6].

Although FD-SAFT significantly improves the quality of the image as compared to processed data, it has poor range resolution. Consequently, small scatterers are not detectable above the background noise.

\section{B. Wide-Band Microwave Holography}

The second method used is wide-band microwave holography referred to as M-HOL in figure captions [6]. It is similar in concept to FD-SAFT, and it also uses angular spectrum decomposition. The raw data measured at the plane $z=0$ is contained in:

$$
g(x, y, \omega)
$$

such that:

$$
0 \leq x \leq x_{\max } \text { and } 0 \leq y \leq y_{\max }
$$

and such that $g$ is sampled at discrete locations in $x$ and $y$ and at discrete frequencies, $\omega$. The goal is to transform the raw data to the 3D holographic representation:

$$
s(x, y, z)
$$

The first step is to decompose the data onto a plane wave spectrum as before, and this must be done independently for every frequency using the 2D Fast Fourier transform:

$$
G\left(k_{x}, k_{y}, \omega\right)=F F T_{2 D}\{g(x, y, \omega)\}
$$


Again, the ranges for $k_{x}$ and $k_{y}$ are centered about zero. Using the dispersion relation:

$$
k_{x}^{2}+k_{y}^{2}+k_{z}^{2}=(2 k)^{2}=\left(2 \frac{\omega}{c}\right)^{2}
$$

one can relate $k_{z}$ to $\omega$ such that:

$$
k_{z}=k_{z}(\omega)=\sqrt{\left(2 \frac{\omega}{c}\right)^{2}-k_{x}^{2}-k_{y}^{2}}
$$

where imaginary values are ignored. This results in the following dataset:

$$
G\left(k_{x}, k_{y}, k_{z}\right)
$$

However, the spacing of $k_{z}$ is not uniform after this transformation and the dataset must be resampled to a uniform distribution of $k_{z}$ using interpolation methods. A fast linear interpolation scheme was used in this investigation. After the necessary resampling is performed the dataset is now:

$$
G^{\prime}\left(k_{x}, k_{y}, k_{z}\right)
$$

To attain the processed 3D holographic representation, $s$, simply an inverse 3D Fast Fourier transform must be performed:

$$
s(x, y, z)=F F T_{3 D}^{-1}\left\{G^{\prime}\left(k_{x}, k_{y}, k_{z}\right)\right\}
$$

The spatial resolution is defined as before, however, the wavelength corresponding to the center frequency is used. Additionally, microwave holography has the benefit of having relatively fine range resolution or resolution along the $\mathrm{z}$-axis, which is defined as:

$$
\delta_{z} \approx \frac{c}{2 B}
$$

where $B$ is the frequency bandwidth of the measurement and $c$ is the speed of light.

\section{RESULTS}

\section{A. SOFI Slab Panels}

Arrangement 1 was the case of the SOFI slab with holes laid face down on the aluminum substrate, as shown in Figure $2 b$. Figure $4 a$ shows the data after processing with FD-SAFT where four of the five holes can be distinguished. FD-SAFT was brought into focus $76 \mathrm{~mm}$ from the measurement plane.
The only hole that cannot be seen is the $3 \mathrm{~mm}$-deep hole. Rather than the signal level from the $3 \mathrm{~mm}$ deep hole dropping below the noise of the system it may be that the edge effects dominated the reflection for this hole and masked it. Edge effects are the result of the edge of the metal plate, and they are more dramatic for edges cross-polarized to the incident wavefront. They are evident in Figure $4 \mathrm{a}$ as vertical lines superimposed on the image. The signal level associated with the $25 \mathrm{~mm}$-deep hole on the right is strong enough to overcome the edge effects, however the signal level from the $3 \mathrm{~mm}$-deep hole on the left is not. The results for microwave holography can be seen in Figure $4 \mathrm{~b}$. A slice of the hologram at $74 \mathrm{~mm}$ shows that all five holes can be distinguished. The $3 \mathrm{~mm}$ deep and can be distinguished better after contrast enhancement (not shown). It also tends to be masked by edge effects occurring near the left edge. However, in comparison, edge effects are far less pronounced in Figure $4 \mathrm{~b}$ than Figure $4 \mathrm{a}$ due to the frequency swept nature of the method.

Arrangement 2 is where the SOFI slab with holes is backed by the blank slab and the substrate, as shown in Figure 2c. The FD-SAFT processed data, seen in Figure 4c, only shows the deepest hole when focused at $76 \mathrm{~mm}$. Edge effects are also visible especially on the left. It is important to note that only the backing was changed between this arrangement and arrangement 1 . It was expected that the results of the two arrangements would be similar, but they are not, and three of the previously detectable holes were not detected in arrangement 2. Therefore, the detection of the holes is not indicative of the reflection from the first SOFI-toair boundary corresponding to the top of the hole. The signal level from that reflection is too low to detect. Furthermore, detection may be more indicative of a phase offset of the wave traveling through SOFI and air as opposed to SOFI alone. This has an effect of changing the apparent depth of the substrate from the measurement plane. This is more evident in the hologram. A slice of the hologram at $76 \mathrm{~mm}$ showed no indication of the holes (not shown). However, a slice of the hologram at $152 \mathrm{~mm}$ corresponding to the level of the substrate revealed indications of four of the five holes as shown in Figure 4d although the holes are now blurred.

Arrangement 3 is when the SOFI slab with holes was sandwiched by the blank slab and the aluminum substrate. This effectively moved the holes away an additional $70 \mathrm{~mm}$ below SOFI as compared to arrangement 1. For this experiment, the power of the received signal was near the noise floor of the particular network analyzer. The power was so low that FD-SAFT processing focused at the level of the substrate $(152 \mathrm{~mm})$ provided no indication of the holes, as shown in Figure 4e. For a single frequency with no averaging, the noise seemed to overwhelm the relatively low level signal from the holes. In comparison, a slice of the hologram at $152 \mathrm{~mm}$ shows the four deepest of the five holes, as shown in Figure $4 \mathrm{f}$. This remarkable increase in target recognition is due to the fact that microwave holography has range resolution to compress the signal from a scatterer along the $z$-direction thereby strengthening the signal greatly above 


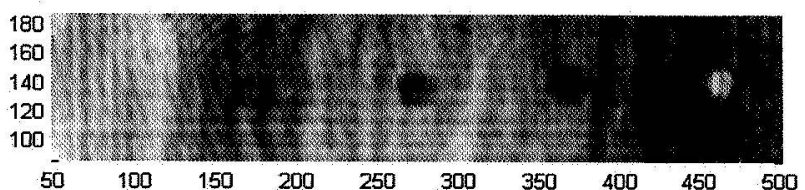

(a)

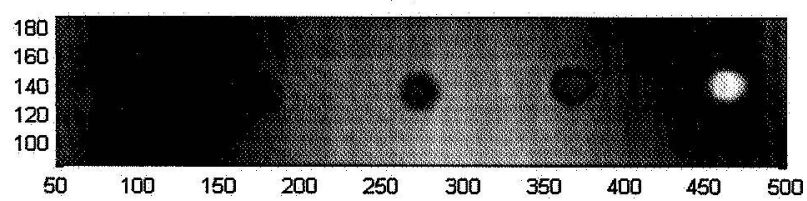

(b)

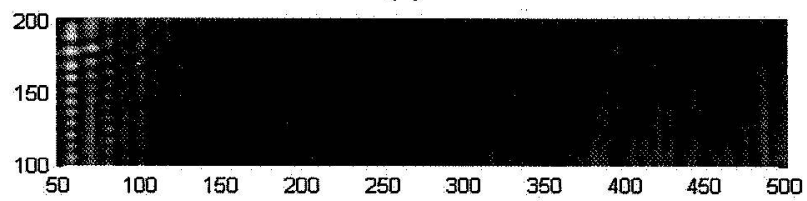

(c)

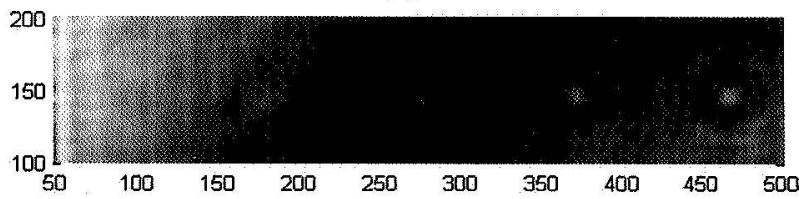

(d)

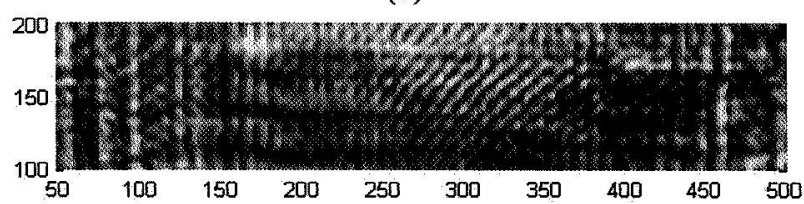

(e)

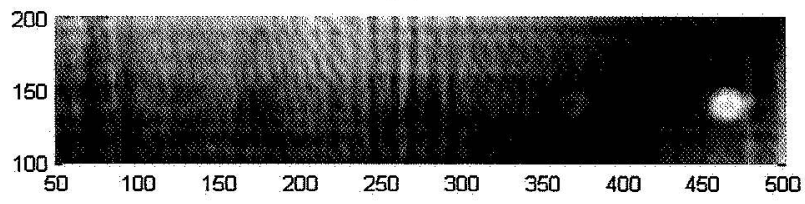

(f)

Fig 4. Flat-bottom hole sample: (a) case 1 FD-SAFT $74 \mathrm{~mm}$, (b) case $1 \mathrm{M}$-HOL $74 \mathrm{~mm}$, (c) case 2 FD-SAFT $74 \mathrm{~mm}$, (d) case $2 \mathrm{M}$-HOL 152 $\mathrm{mm}$, (e) case 3 FD-SAFT $152 \mathrm{~mm}$, and (f) case $3 \mathrm{M}-\mathrm{HOL} 152 \mathrm{~mm}$.

noise. Also, noise is further suppressed by summing over the frequency and consequently averaging noise out.

\section{B. Multiple Flat-Bottom Hole SOFI Test Panel}

Results from the test panel as illustrated in Figure 3 make it possible to determine the dimensions of the smallest detectable air void in SOFI given the previously defined measurement setup. The FD-SAFT processed data for this panel is shown in Figure 5a where FD-SAFT was focused at $110 \mathrm{~mm}$ from the measurement plane to provide a result with the least noise. The location of the holes were known a priori so holes were determined to be distinguished so long as they locally disturbed the image. The smallest diameter detected was $13 \mathrm{~mm}$ and the shallowest depth was $13 \mathrm{~mm}$. The

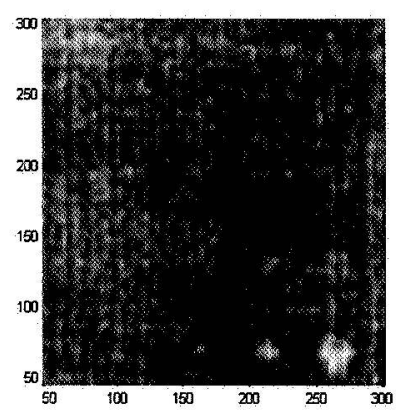

(a)

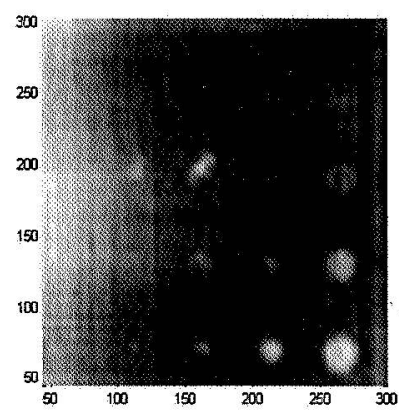

(b)
Fig 5. Processed images for the test panel: (a) FD-SAFT and (b) microwave holography.

smallest volume detected was the $13 \mathrm{~mm}$-diameter and 19 mm-deep hole corresponding approximately to a volume of $2.4 \mathrm{~cm}^{3}$. A slice of the hologram at $110 \mathrm{~mm}$ from the measurement plane shows the holes much better, as shown in Figure 5b. Compared to Figure 5a, the image shown in Figure $5 \mathrm{~b}$ has a lower noise level and more holes can be detected. The smallest hole detected was $6 \mathrm{~mm}$ in diameter and $6 \mathrm{~mm}$ deep corresponding to a volume of $0.2 \mathrm{~cm}^{3}$, which is much smaller than the smallest hole detected by FD-SAFT.

\section{SUMMARY}

Defects like air void in SOFI can significantly reduce its integrity. Microwave NDE methods using synthetic focusing at relatively low frequency have shown comparable results to real focusing methods operating at much higher frequency $[2,3]$. The performance of both image processing methods is very quick requiring only a fraction of a second to process the FD-SAFT method and only a few seconds to process the microwave holography method. The same image processing methods will soon be applied at higher frequencies such as Ka-Band from 26.5 to $40 \mathrm{GHz}$ and V-band from 50 to 75 GHz. These higher frequency bands will offer increased spatial and range resolutions to detect even smaller defects.

\section{ACKNOWLEDGEMENT}

Funding for this work was provided by the NASA Marshall Space Flight Center through a Cooperative Agreement.

\section{REFERENCES}

[1] Columbia Accident Investigation Board Report, NASA, August 2003.

[2] S. Kharkovsky, F. Hepburn, J. Walker, R. Zoughi. "Nondestructive Testing of the Space Shuttle External Tank Foam Insulation using Near-Field and Focused Millimeter Wave Techniques," Materials Evaluation 63, N5, 516-522 (2005).

[3] S. Shrestha, S. Kharkovsky, R. Zoughi and F. Hepburn. "Microwave and Millimeter Wave Nondestructive Testing of the Space Shuttle External Tank Insulating Foam," Materials Evaluation 63, N3, 339-344 (2005). 
[4] L.J. Busse, "Three-Dimensional Imaging Using a Frequency-Domain Synthetic Aperture Focusing Technique," IEEE Transactions on Ultrasonics, Ferroelectrics, and Frequency Control 39, No. 2, 174-179 (1992).

[5] J.T. Case, J. Robbins, S. Kharkovsky, F. Hepburn, and R. Zoughi, "Microwave And Millimeter Wave Imaging of the Space Shuttle External Fuel Tank Spray On Foam Insulation (SOFI) Using Synthetic Aperture Focusing Techniques (SAFT)," To appear in the Proceedings of the Thirty-second Annual Review of Progress in Quantitative Nondestructive Evaluation, (2006).

[6] D.M. Sheen, D.L. McMakin, T.E. Hall, "Three-Dimensional Millimeter-Wave Imaging for Concealed Weapon Detection," IEEE Transactions on Microwave Theory and Techniques 49, No. 9, (2001).

[7] J.W. Goodman, Introduction to Fourier Optics, New York: McGrawHill, 1968.

[8] F.T. Ulaby, R.K. Moore and A.K. Fung, "Microwave Remote Sensing, Active and Passive" Vol. II, Artech House, Norwood, MA, 1986.

[9] Ahmed, Yamani, "Three-Dimensional Imaging Using a New Synthetic Aperture Focusing Technique," IEEE Transactions on Ultrasonics, Ferroelectrics, and Frequency Control 44, No. 4, (1997). 